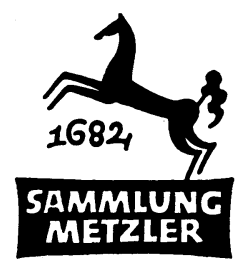

REALIEN ZUR LITERATUR

ABT. D:

LITERATURGESCHICHTE 


\section{Gerhart Hauptmann}

2., durchgesehene und ergänzte Auflage

ERSCHIENEN IM DREIHUNDERTSTEN JAHR DER J. B. METZLERSCHE VERLAGSBUCHHANDLUNG STUTTGART 


\author{
1. Aufl. 1974 (1.- 5. Tsd.) \\ 2. Aufl. 1982 (6.-10. Tsd.)
}

CIP-Kurztitelaufnahme der Deutschen Bibliothek

\title{
Hoefert, Sigfrid:
}

Gerhart Hauptmann/Sigfrid Hoefert. - 2., durchges.

u. erg. Aufl. - Stuttgart: Metzler, 1982.

(Sammlung Metzler; M 107: Abt. D, Literaturgeschichte)

ISBN 978-3-476-12107-3

NE: GT

ISBN 978-3-476-12107-3

ISBN 978-3-476-04085-5 (eBook)

DOI 10.1007/978-3-476-04085-5

\section{107}

() 1982 Springer-Verlag GmbH Deutshland Ursprünglich erschienen bei J.B. Metzlersche Verlagsbuchhandlung und Carl Ernst Poeschel Verlag GmbH in Stuttgart 1974/1982 
Abkürzungen. ................. VI

Vorbemerkungen .................. IX

Vorbemerkung zur 2. Auflage $\ldots \ldots \ldots \ldots \ldots \ldots$ X

Abgekürzt zitierte Literatur . . . . . . . . . . . . XI

I. Handschriften, Selbstzeugnisse, Ausgaben und Bibliographien .................... 1

II. Leben und Werk Hauptmanns . . . . . . . . . . 6

1. Jugend und schöpferische Anfänge (1862-1889) . . . 6

2. Die Entwicklung bis zur Jahrhundertwende (1889-1900)....................... 16

3. Von der Jahrhundertwende bis zum 1. Weltkrieg (1900-1914)....................... 37

4. Vom 1. Weltkrieg bis zum Ende der Republik (1914-1933)...................... 55

5. Die Jahre der NS-Diktatur (1933-1945) . . . . . . 71

6. Ausklang (1945/46) . . . . . . . . . . . . 85

III. Stand und Aufgaben der Forschung . . . . . . . 91

Personenregister. . . . . . . . . . . . . . . . 121

Werkregister ..................... 134 


\section{ABKüRZUNGEN}

$\begin{array}{ll}\text { ABI } & \text { 8-Uhr-Abendblatt } \\ \text { AG } & \text { Acta Germanica } \\ \text { AG et R } & \text { Acta Germanica et Romanica } \\ \text { AGR } & \text { American German Review } \\ \text { ASf } & \text { Archiv für Sippenforschung } \\ \text { ASG } & \text { Annali Sezione Germanica } \\ \text { ASSL } & \text { Archiv für das Studium der neueren Sprachen und Literaturen } \\ \text { AUP } & \text { The Australian Goethe Society Proceedings } \\ \text { BB } & \text { Berliner Börsen-Courier } \\ \text { BDB } & \text { Börsenblatt für den deutschen Buchhandel (Frankfurter Aus- } \\ & \text { gabe) } \\ \text { BDTh } & \text { Blätter des Deutschen Theaters } \\ \text { BL } & \text { Berliner Lokalanzeiger } \\ \text { Bresl. } & \text { Breslauer } \\ \text { BT } & \text { Berliner Tageblatt } \\ \text { BZ } & \text { Breslauer Zeitung } \\ \text { CA } & \text { Centenar-Ausgabe } \\ \text { DB } & \text { Doitsu Bungaku } \\ \text { DNN } & \text { Dresdener Neueste Nachrichten } \\ \text { DR } & \text { Deutsche Rundschau } \\ \text { DU } & \text { Der Deutschunterricht } \\ \text { DVjs } & \text { Deutsche Vierteljahrsschrift für Literatur und Geistes- } \\ & \text { geschichte } \\ \text { EG } & \text { Etudes Germaniques } \\ \text { ER } & \text { Europäische Revue } \\ \text { EUPH } & \text { Euphorion } \\ \text { FB } & \text { Freie Bühne } \\ \text { FMLS } & \text { Forum for Modern Language Studies } \\ \text { FZ } & \text { Frankfurter Zeitung } \\ \text { GES } & \text { Die Gesellschaft } \\ \text { Ges. } & \text { Gesellschaft } \\ \text { GGA } & \text { Göttingische Gelehrte Anzeigen } \\ \text { GHJ } & \text { Gerhart Hauptmann-Jahrbuch } \\ \text { GLL } & \text { German Life and Letters } \\ \text { GQ } & \text { German Quarterly } \\ \text { GR } & \text { Germanic Review } \\ \text { GRM } & \text { Germanisch-Romanische Monatsschrift } \\ \text { GSJ } & \text { Greifswald-Stralsunder Jahrbuch } \\ \text { HF } & \text { Hamburger Fremdenblatt } \\ \text { IR } & \text { Das innere Reich } \\ \text { JbBK } & \text { Jahrbuch für Berlin-Brandenburgische Kirchengeschichte } \\ \text { JbDS } & \text { Jahrbuch der deutschen Schillergesellschaft } \\ \text { JbDSh } & \text { Jahrbuch der deutschen Shakespeare-Gesellschaft } \\ \text { JbSB } & \text { Jahrbuch der Schlesischen Friedrich-Wilhelms-Universität zu } \\ & \text { Breslau } \\ \end{array}$




$\begin{array}{ll}\text { JbSPK } & \text { Jahrbuch der Stiftung Preußischer Kulturbesitz } \\ \text { JEGP } & \text { Journal of English and Germanic Philology } \\ \text { JJQ } & \text { James Joyce Quarterly } \\ \text { JR } & \text { Jahresring } \\ \text { KN } & \text { Kwartalnik Neofilologiczny } \\ \text { KNS } & \text { Korrespondenzblatt des Vereins für niederdeutsche Sprach- } \\ & \text { forschung } \\ \text { Kw } & \text { Kunstwart } \\ \text { LE } & \text { Das literarische Echo } \\ \text { LN } & \text { Literaturnoje nasledstwo } \\ \text { LW } & \text { Die literarische Welt } \\ \text { MAG } & \text { Das Magazin für Literatur } \\ \text { MGS } & \text { Michigan Germanic Studies } \\ \text { MH } & \text { Monatshefte für deutschen Unterricht } \\ \text { Mittln. } & \text { Mitteilungen } \\ \text { Mk } & \text { Merkur } \\ \text { MLJ } & \text { Modern Language Journal } \\ \text { MLN } & \text { Modern Language Notes } \\ \text { MLQ } & \text { Modern Language Quarterly } \\ \text { MLR } & \text { Modern Language Review } \\ \text { MNN } & \text { Münchener Neueste Nachrichten } \\ \text { MPh } & \text { Modern Philology } \\ \text { MuK } & \text { Maske und Kothurn } \\ \text { MWBG } & \text { Mitteilungen der Wilhelm-Busch-Gesellschaft } \\ \text { Nachr. } & \text { Nachrichten } \\ \text { NDH } & \text { Neue Deutsche Hefte } \\ \text { NDL } & \text { Neue Deutsche Literatur } \\ \text { NDR } & \text { Neue Deutsche Rundschau } \\ \text { NFP } & \text { Neue Freie Presse } \\ \text { NGS } & \text { New German Studies } \\ \text { NLZ } & \text { Neue Leipziger Zeitung } \\ \text { NPh } & \text { Neophilologus } \\ \text { NR } & \text { Neue Rundschau } \\ \text { NWJ } & \text { Neues Wiener Journal } \\ \text { NWT } & \text { Neues Wiener Tageblatt } \\ \text { OL } & \text { Orbis Litterarum } \\ \text { OMh } & \text { Ostdeutsche Monatshefte } \\ \text { PJ } & \text { Preußische Jahrbücher } \\ \text { PK } & \text { Preußischer Kulturbesitz } \\ \text { PL } & \text { Poet Lore } \\ \text { PMLA } & \text { Publicatiuons of the Modern Language Association of } \\ & \text { America } \\ \text { PP } & \text { Pädagogische Provinz } \\ \text { PQ } & \text { Philological Quarterly } \\ \text { RA } & \text { Revue d'Allemagne } \\ \text { RLC } & \text { Revue de Littérature comparee } \\ \text { RLV } & \text { Revue des Langues vivantes } \\ \text { RR } & \text { Romanic Review } \\ & \end{array}$




$\begin{array}{ll}\text { schl. } & \text { schlesisch } \\ \text { SdMh } & \text { Süddeutsche Monatshefte } \\ \text { SMh } & \text { Sozialistische Monatshefte } \\ \text { StN } & \text { Studia Neophilologica } \\ \text { SuF } & \text { Sinn und Form } \\ \text { SwMh } & \text { Schweizer Monatshefte } \\ \text { Tl. } & \text { Teil } \\ \text { UTQ } & \text { The University of Toronto Quarterly } \\ \text { VB } & \text { Völkischer Beobachter } \\ \text { VZ } & \text { Vossische Zeitung } \\ \text { WB } & \text { Weimarer Beiträge } \\ \text { WMh } & \text { Westermanns Monatshefte } \\ \text { WW } & \text { Wirkendes Wort } \\ \text { WZUH } & \text { Wissenschaftliche Zeitschrift der Martin-Luther-Universität } \\ & \text { Halle-Wittenberg } \\ \text { ZB } & \text { Zentralblatt für Bibliothekswesen } \\ \text { ZDP } & \text { Zeitschrift für deutsch e Philologie } \\ \text { ZfDB } & \text { Zeitschrift für deutsche Bildung } \\ \text { ZfDk } & \text { Zeitschrift für Deutschkunde } \\ \text { ZfSl } & \text { Zeitschrift für Slawistik } \\ \text { Zs. } & \text { Zeitschrift }\end{array}$




\section{VORBEMERKUNG}

Die vorliegende Arbeit will dem Forschenden eine schnelle und zuverlässige Orientierung ermöglichen; sie setzt sich zum Ziel, den Werdegang Gerhart Hauptmanns zu beleuchten, in sein dichterisches Schaffen einzuführen sowie einen Überblick über das Schrifttum zu geben, das über ein gewisses Problem oder einen Problemkreis, ein einzelnes Werk oder eine Werkgruppe vorliegt. Gemäß der Ausrichtung der 'Sammlung Metzler ' wird auf die Darbietung von Daten, Fakten und den jeweils erreichten Forschungsergebnissen besonderer Wert gelegt. Angesichts der vielschichtigen Fülle des Materials werden vor allem solche Arbeiten aufgenommen, die das Verständnis des Hauptmannschen Werkes fördern, seine Breite und Vielfalt klären helfen oder sonst aus historischer Sicht von Bedeutung sind. Es wird in den Literaturangaben eher zuviel als zuwenig dargeboten; in Zweifelsfällen wurde immer für die Aufnahme der betreffenden Arbeit entschieden. Weitere Hilfsmittel bieten sich dem Suchenden in den verschiedenen Bibliographien dar.

Was die Anlage des vorliegenden Bandes betrifft, so werden im ersten Teil Bemerkungen zur Handschriftensituation, zu den Ausgaben und bibliographischen Arbeiten dargeboten. Im zweiten Teil wird die Entwicklung Hauptmanns in chronologischer Reihenfolge und gedrängter Kürze in sechs großen Abschnitten gezeigt; am Ende eines jeden Abschnitts bringen die bibliographischen Informationen neben den rein technischen Angaben Hinweise auf aufschlußreiche biographische Zeugnisse sowie auf das notwendige Schrifttum zu den jeweils besprochenen Werken. Auch erfolgt eine Zusammenstellung der Reden, Aufsätze und Fragmente des betreffenden Zeitraumes. Im dritten Teil wird der Forschungsstand im Hinblick auf die verschiedenen Probleme und Problemkreise erörtert und der Aufgabenbereich der Hauptmann-Forschung umrissen.

Wertvolle Hilfeleistungen wurden mir beim Zustandekommen dieser Arbeit vor allem von Dr. W. Dittrich und Herrn R. Ziesche (Staatsbibliothek Berlin), Herrn A. Dreifuß (Märkisches Museum), Herrn A. Münch (Hauptmann-Archiv Radebeul) sowie meinen Kollegen Dr. M. Richter und Dr. G. Brude-Firnau zuteil. Ihnen gilt mein besonderer Dank. Auch bin ich dem Canada Council für die mir gewährte finanzielle Unterstützung zu Dank verpflichtet.

Waterloo/Ontario

Sigfrid Hoefert 
Für die Neuauflage des vorliegenden Bandes wurden Text und Literaturangaben durchgesehen und auf den neuesten Stand gebracht. Berücksichtigt wurden Arbeiten, die bis Sommer 1981 veröffentlich und erhältlich waren. Um die Fülle der bibliographischen Gegebenheiten auf ein annehmbares Maß zurückzuführen, wurden besonders die Literaturangaben älteren Datums kritisch gesichtet und reduziert. Allerdings ist der Umfang der bibliographischen Hinweise dadurch kaum vermindert worden. Die neue Literatur ist umfangreich; sie vermittelt Perspektiven, die nicht nur für den Hauptmannforscher relevant sein dürften.

Waterloo/Ontario

Sigfrid Hoefert 


\section{ABgEKÜRZT ZITIERTE LITERATUR}

Alexander, $1964=$ N. E. Alexander, Studien zum Stilwandel im dramatischen Werk G.H's, 1964.

Bebl, 1948 = C. F. W. Behl, Wege zu G.H., 1948.

Bleich, 1936 = E. H. Bleich, Der Bote aus der Fremde als formbedingender Kompositionsfaktor im Drama des Naturalismus. (Ein Beitrag zur Dramaturgie des Naturalismus), Diss. Greifswald 1936.

Bleicker, 1961 = W. Bleicker, Formen des Gesprächs im Prosawerk G.H's, Diss. Mainz 1961.

Brahm, $1913=$ O. Brahm, Kritische Schriften über Drama und Theater, hrsg. v. P. Schlenther, 1913.

Brammer, 1972 = U. G. Brammer, Selbstbildnis in G.H's Dramen, Ph. D. Diss. University of Pittsburgh 1972.

Centenary Lectures, 1964 = Hauptmann Centenary Lectures, hrsg. v. K. S. Knight und F. Norman, London 1964.

Dill, 1972 = W. O. Dill, Der Dionysosmythos als Strukturelement in G. H's Prosa, Ph. D. Diss. University of California 1972.

Dosenbeimer, $1967=\mathrm{E}$. Dosenheimer, Das deutsche soziale Drama von Lessing bis Sternheim, 1967.

Fecbter, 1922 = P. Fechter, G.H., 1922.

Fiedler, $1954=$ R. Fiedler, die späten Dramen G.H's. Versuch einer Deutung, 1954.

Fischer, 1957 = G. Fischer, Erzählformen in den Werken G.H's, 1957.

Gregor, 1951 = J. Gregor, G.H. Das Werk und unsere Zeit, 1951.

Gutbke, 1961 = K. S. Guthke, G.H. Weltbild und Werk, 1961, ${ }^{2} 1980$ (überarb.).

Hauptmann, 1942 = G.H. zum 80. Geburtstag am 15. November 1942, 1942.

Heise, 1923 = W. Heise, G.H., 4 Bde., 1923.

Heynen, 1922 = Mit G.H.: Erinnerungen und Bekenntnisse aus seinem Freundeskreis, hrsg. v. W. Heynen, 1922.

Hensel, $1957=$ M. Hensel, Die Gestalt Christi im Werk G.H's, Diss. Berlin (FU) 1957.

Heuser, 1961 = F. W. J. Heuser, G.H. Zu seinem Leben und Schaffen, 1961.

Hilscher, 1969 = E. Hilscher, G.H., 1969, ${ }^{2} 1974,{ }^{3} 1979$ (überarb.).

Hortenbach, $1965=\mathrm{J}$. C. Hortenbach, Freiheitsstreben und Destruktivität. Frauen in den Dramen A. Strindbergs und G.H's, Oslo 1965.

Hurtig, 1956 = G. Hurtig, Die Lichtsymbolik bei G.H., Diss. Marburg 1956.

Kerr, 1917 = A. Kerr, Die Welt im Drama, 5 Bde., 1917.

Kersten, 1966 = G. Kersten, G.H. und L. N. Tolstoi. Studien zur Wirkungsgeschichte von L. N. Tolstoi in Deutschland 1885-1910, 1966.

Krause, 1952 = E. Krause, G.H's frühe Dramen im Spiegel der Kritik, Diss. Erlangen 1952.

Künzel, $1962=\mathrm{H}$. Künzel, Die Darstellung des Todes in den Dramen G.H's und G. Kaisers, Diss. Erlangen 1962. 
Langer, 1932 = L. Langer, Komik und Humor bei G.H., Diss. Kiel 1932.

Leiner, 1955 = F. Leiner, Der Gedanke der Wiedergeburt im Leben und Werk G.H's, Diss. München 1955.

Liebenstein, 1950 = W. Liebenstein, G.H. und das Reformationszeitalter, Diss. München 1950.

Lindner, 1949 = A. Lindner, Das Alterswerk G.H's. Versuch einer Deutung, Diss. Wien 1949.

Machatzke, 1963 = G.H. Die Kunst des Dramas. Über Schauspiel und Theater, zusammengest. v. M. Machatzke, 1963.

Marcuse, 1922 = G.H. und sein Werk, hrsg. v. L. Marcuse, 1922.

Mehring, 1961 = F. Mehring, Aufsätze zur deutschen Literatur von Hebbel bis Schweichel, hrsg. v. H. Koch, 1961.

Meixner, $1961=$ H. Meixner, Naturalistische Natur: Bild und Begriff der Natur im naturalistischen Drama, Diss. Freiburg i. Br. 1961.

Mendelssobn, 1970 = P. de Mendelssohn, S. Fischer und sein Verlag, 1970.

Metken, 1954 = G. Metken, Studien zum Sprachgestus im dramatishen Werk G.H's, Diss. München 1954.

Michaelis, 1962 = R. Michaelis, Der schwarze Zeus. G.H's zweiter Weg, 1962.

Müller, 1939 = I. Müller, G.H. und Frankreich, 1939.

Muller, 1950 = S. H. Muller, G.H. und Goethe, 1950 (auf engl. 1949).

Osborne, 1971 = J. Osborne, The Naturalist Drama in Germany, Manchester 1971.

Requardt, 1955 = W. Requardt, Erkner im Leben und Werk G.H's unter besonderer Berücksichtigung der Novelle »Fasching «, Diss. Hamburg 1955.

Requardt/Machatzke, $1980=$ W. Requardt und M. Machatzke, G.H. und Erkner. Studien zum Berliner Frühwerk, 1980.

Robmer, 1958 = R. Rohmer, Die Romane G.H's, Diss. Leipzig 1958.

Schreiber, $1946=$ H. Schreiber, G.H. und das Irrationale, 1946.

Schrimpf, $1963=$ H. J. Schrimpf, Struktur und Metaphysik des sozialen Schauspiels bei G.H., in: Literatur und Gesellschaft vom neunzehnten ins zwanzigste Jahrhundert, Festgabe für B. v. Wiese, hrsg. v. H. J. Schrimpf, 1963, S. 274-308.

Shaw, 1958 = L. R. Shaw, Witness of Deceit. G.H. as Critic of Society, Berkely u. Los Angeles 1958.

Sinden, 1957 = M. Sinden, G.H. The Prose Plays, Toronto 1957.

Steffen, $1964=\mathrm{H}$. Steffen, Figur und Vorgang im naturalistischen Drama G.H's, in: DVjs, 38 (1964), S. 424-449.

Taube, 1936 = G. Taube, Die Rolle der Natur in G.H's Gegenwartswerken bis zum Anfang des 20. Jahrhunderts, 1936.

Tettenborn, $1950=$ J. Tettenborn, Das Tragische bei G.H., Diss. Jena 1950.

Thielmann, $1937=\mathrm{H}$. Thielmann, Stil und Technik des Dialogs im neueren Drama. (Vom Naturalismus bis zum Expressionismus), Diss. Heidelberg 1937.

Van der Will, 1962 = W. van der Will, Voraussetzungen und Möglichkeiten einer Symbolsprache im Werke G.H's, Diss. Köln 1962. 
Voigt, 1965 = F. A. Voigt, G.H. und die Antike, hrsg. v. W. Studt, 1965. Vollmers-Schulte, 1923 = F. Vollmers-Schulte, G.H. und die soziale Frage, 1923.

Weisert, $1949=$ J. J. Weisert, The Dream in G.H., New York 1949. Zimmermann, $1964=$ R. C. Zimmermann, Die Pathetik des heiligen Berstens und ihre Gestaltwandlungen im Werk G.H's, in: Formenwandel, Festschrift zum 65. Geburtstag von P. Böckmann, hrsg. v. W. Müller-Seidel u. W. Preisendanz, 1964, S. 426-470. 